\title{
Relationships between Upper Level Circulation over South America and Rainfall over South Eastern South America: A Physical Base for Seasonal Predictions
}

\author{
Laura Zamboni \\ Dipartimento di Matematica, Universitá degli Studi di Trieste, Italy \\ The Abdus Salam, International Centre for Theoretical Physics, Italy \\ Dept. of Atmospheric and Oceanic Sci., University of California Los Angeles, USA \\ Carlos R. Mechoso \\ Dept. of Atmospheric and Oceanic Sci., University of California Los Angeles, USA \\ Fred Kucharski \\ The Abdus Salam, International Centre for Theoretical Physics, Italy
}




\begin{abstract}
The existence of a significant simultaneous correlation between bi-monthly mean precipitation anomalies over South Eastern South America (SESA) and either the first or the second (depending on season) leading mode of interannual variability of upper level wind over South America (SA) is demonstrated in all seasons, except in winter. The pattern associated with these modes of variability is similar in all seasons, and consists of a continental-scale vortex centered over the eastern coast of subtropical SA. The vortex has a quasi-barotropic structure in all seasons and its variability modifies moisture transport from the South American Low Level Jet (SALLJ) and the western tropical Atlantic to SESA thus creating precipitation anomalies in this region. In spring (October-November) and summer (January-February) the circulation creates a second center of precipitation anomalies over the South Atlantic Convergence Zone (SACZ), which are of opposite sign to those over SESA; while in fall (April-May) precipitation anomalies are primarily confined to SESA.
\end{abstract}

On the basis of the correlation between upper level winds and precipitation, an empirical method to produce long-range forecasts of bi-monthly mean precipitation over SESA is developed. Method tests' in hindcast mode for the period 1959 - 2001 show a potential for reliable predictions in the southern spring, summer and fall. The method is further tested in an experimental mode by using DEMETER winds' hindcasts. Forecasts obtained in this way are skillful in spring only, with highest skill in El Niño Southern Oscillation (ENSO) years. In 
summer and fall, the DEMETER forecasts of wind anomalies limit the method's capability on making reliable real predictions.

\section{INTRODUCTION}

Reliable climate forecasts help decision-makers in the planning of effective responses to departures from the mean seasonal cycle (seasonal anomalies) in anticipation of their occurrence. Precipitation anomalies can have strong impacts on human activities, especially in regions strongly dependent upon agriculture and hydroelectricity production such as La Plata River's basin in South Eastern South America (SESA). In SESA, climatological precipitation has small seasonal variations (e.g. Montecinos et al. 2000). Summer and spring precipitation is part of the South American Monsoon System, whereas fall and winter precipitation originates from synoptic weather systems. Seasonal precipitation anomalies over SESA have a dominant pattern of uniform sign over the region, and strong interannual variations with values ranging from 50 to $150 \%$ of the mean (Fig.1). The SESA anomalies form the southern component of an approximately north-south dipole of precipitation anomalies over South America (SA) during spring and summer (e.g. Casarin and Kousky 1986; Paegle and Mo 1997; Paegle et al. 2002; Robertson and Mechoso 2000; Diaz and Aceituno 2003). The dipole variability has been linked to anomalies in other outstanding climate features such as the intensity of the South Atlantic Convergence Zone (SACZ), South American Low Level Jet (SALLJ), Pacific-South American (PSA) modes, and El Niño/Southern Oscillation (ENSO). Several papers extensively document such links (e.g., Robertson and Mechoso 2000; Díaz and Aceituno 2003; Mo and Paegle 2001; 
Paegle and Mo 2002; Liebmann et al. 2004, Ferreira et al. 2003, Grimm 2003, Grimm et al. 2007, Silva et al. 2009 ).

During the austral spring ENSO is significantly and positively correlated with rainfall variability in subtropical SA (Montecinos et al. 2000); this relationship serves as the foundation for interannual predictions of rainfall in that region. The different phases of ENSO (El Niño and La Niña) tend to generate precipitation anomalies with opposite sign, which indicates a large degree of linearity in the teleconnection with the Tropical Pacific Ocean (Grimm et al. 2000).

ENSO impacts are found during summer as well, even though with weaker intensity. It has been suggested that the effects of the South American monsoon system predominate in summer over those associated with remote forcings (e.g. Cazes-Boezio et al. 2003; Grimm, 2003). Paegle and Mo (2002) described ENSO's impacts on SA during summer by means of EOF analysis of rainfall, and found anomalies of opposite sign over Northeastern Brazil and SESA. Cazes-Boezio et al. (2003) outlined a dynamical picture of ENSO's impact on the South American climate by EOF analysis of upper level winds and regression maps of the leading principal components (PCs) over different fields. Their results show the leading mode of upper level variability over SA during spring to be part of a wave pattern that is highly positively correlated with SSTs in the Tropical Pacific (up to 0.6 in the El Niño 3.4 region). The pattern is also significantly and positive correlated with anomalies in the vertical velocity (omega) over northeastern SA, and negative correlated with those over SESA. (A consistent result is obtained, by the same authors, for the mean value of rainfall anomalies obtained by 13 rainfall gauge stations over Uruguay.) Cazes-Boezio et al. (2003) proposed that the interannual variability of upper level wind can be 
interpreted as changes in the frequency of occurrence of intraseasonal circulation regimes over the South Pacific, which would be triggered by ENSO in spring and would originate from nonlinear processes in fall-winter. A different perspective is that Rossby wave propagation from the central and western equatorial Pacific contributes to the development of the leading PSA (PSA1) in all seasons (Mo 2000; Mo and Paegle 2001). Precipitation anomalies over SA have been interpreted as downstream effects of the PSA1 and PSA2 modes (Mo and Paegle 2001) in summer and spring respectively; they did not investigate similar relationship for precipitation over SA during the fall season.

Doyle and Barros (2002) suggested tropical and southwestern Atlantic SST anomalies as source of predictability in summer. Cazes-Boezio et al. (2003) found significant correlations between the $2^{\text {nd }}$ mode of upper level wind variability over SA and rainfall over SESA, and a north-south dipole in the SSTs field over the Southern Atlantic.

Diaz et al. (1998) identified a connection between Atlantic SST anomalies under the oceanic SACZ extension and precipitation over southern Brazil and Uruguay in fall-early winter. In their canonical correlation analysis warm (cold) SST anomalies correspond to wet (dry) rainfall anomalies. Cazes-Boezio et al. (2003) reported the same type of relationship by correlation analysis for the fall-winter season.

This short review demonstrates that various processes have been recognized as modulators of seasonal rainfall over SESA on interannual timescales. Current operational models, however, seem unable to exploit the associated potential for predictability of seasonal precipitation anomalies in the region. For example, the International Research Institute for Climate and 
Society (IRI) provides, since 1997, probabilistic forecasts for below-, near- and above normal for precipitation and temperature, one month in advance for the upcoming 3 months. The forecasts are obtained by combining products of dynamical models and simple empirical prediction methods based on the phases of El Niño-Southern Oscillation (ENSO). The skill of IRI seasonal forecast over subtropical SA, as evaluated by ranked probabilistic skill score (RPSS) with respect to climatology (Wilks 1995), is between -0.1 and 0.1 for all seasons except for the austral spring, when the skill can increase up to 0.3 (Goddard et al. 2003). Starting December 1997, the Climate Outlook Forum (COF) has been producing seasonal precipitation forecasts for SA east of the Andes and between $20 \mathrm{~S}$ and $40 \mathrm{~S}$. The forecasts are obtained by combining outputs of several numerical and statistical models, results of diagnostic analysis, and statistic on the regional climate variability; and are released in the same format as those from IRI. Berri et al. (2005) found that the average RPSS of COF forecasts for all seasons is negative over most of SESA. The European Centre for Medium-Range Weather Forecasts (ECMWF), together with other research and operational centers in Europe, developed the DEMETER (Development of a European Multimodel Ensemble system for seasonal to inTERannual prediction) project in order to assess the capability of a multi general circulation model (GCM) ensemble forecast system to produce seasonal to interannual forecasts (Palmer et al. 2004). The GCMs participating in the project produced hindcasts from 1959 to 2001 . The multimodel ensemble mean hindcast by ECMWF, the United Kingdom Met Office (UKMO) and Météo-France (METF) captures the main pattern and intensity of precipitation climatology over SA in all seasons. Nevertheless, precipitation anomaly forecasts over SESA present small departures from the climatology, and 
most of the observed values fall outside of the $95 \%$ prediction interval as defined by the variance of the ensemble mean. Hindcasts have marginal skill when GCMs are run the same month the prediction targets, as in November-December (ND) and May-June (MJ). Recently, Coelho et al. (2006) have developed an integrated seasonal forecast system that combines an empirical model, which uses Pacific and Atlantic SSTs as predictor of precipitation over SA, and a multimodel coupled system. For the southern summer the method generally produces marginally skillful predictions over SESA (see their Fig.9), while better predictions result in the occurrence of ENSO events (see their Fig.10).

In this study we are interested in identifying other predictors of seasonal precipitation over SESA in all seasons, and in assessing their potential for improved predictions. We start by finding a relationship between interannual variability of precipitation over SESA and of winds at upper levels. Next we provide a physical interpretation of the documented relationship. Finally, we explore how the relationship found can contribute to produce long-range reliable seasonal precipitation forecasts over SESA.

The datasets we use are described in section 2. Section 3 presents the physical relationship between upper level wind and precipitation variability. Section 4 describes our proposed methodology for long range precipitation forecasts and discusses the expected forecast skill. Section 5 includes a summary and concluding remarks.

\section{DATA}


Our main focus is on the region $60-45 \mathrm{~W} ; 24-38 \mathrm{~S}$, which represents SESA (Fig. 1a). For the selected region, we represent seasonal mean precipitation by the domain average over the central two-month periods in each season. This procedure captures the coherent signal throughout each season and filters high frequency variability. The bimonthly periods, therefore, are OctoberNovember (ON), January-February (JF), April-May (AM) and July-August (JA). We investigate the period 1959-2001.

The precipitation dataset we use was compiled by the Climate Research Unit (CRU), University of East Anglia (http://www.cru.uea.ac.uk/cru/data/hrg.htm). In this dataset, values of monthly precipitation were reconstructed from gauge measurements. The fields are available at $0.5^{\circ} \mathrm{x}$ $0.5^{\circ}$ horizontal resolution over land in the period 1901-2002 (New et al. 1999; 2000).

The wind fields at 1000, 925, 850, 700, 600, 500, 400, 300 and $200 \mathrm{hPa}$, latent heat flux and specific humidity correspond to the global reanalysis at the National Centers for Environmental Prediction-National Center for Atmospheric Research (NCEP-NCAR). This dataset is available at $2.5^{\circ} \times 2.5^{\circ}$ horizontal resolution from 1948 to present (Kalnay et al. 1996).

For SSTs we use the National Oceanic and Atmospheric Administration's (NOAA) extended reconstructed SSTs dataset (Smith and Reynolds 2004). In this dataset, monthly mean values are available for the global ocean at $2^{\circ} \times 2^{\circ}$ horizontal resolution, from 1855 to present.

For the tests of our method in a realistic forecast framework, we use the predictions of $200 \mathrm{hPa}$ winds produced by the DEMETER GCMs. The initial conditions for the atmospheric and land states in these predictions were provided by the ECMWF 40-yr Reanalysis (ERA40), four times each year on the $1^{\text {st }}$ day of February, May, August and November. Nine ensemble members of 
six-month long predictions were generated by each GCM by changing the ocean initial conditions (Palmer et al. 2004). Since predictions by different models cover different periods we chose the ECMWF, the United Kingdom Met Office (UKMO) and Météo-France (METF) products, which have the longest overlap and consequently define our period of investigation. The DEMETER data have 2-3 months lag with respect to the initial conditions for the bi-months we considered.

For simplicity, we consider the multimodel ensemble mean over all the ensemble members of the three GCMs, and we will refer to it as "EMU" in the remainder of the text. The advantage of the multimodel approach is to enlarge the variability simulated by each model's physics since the ensemble covers a larger portion of the phase space.

\section{UPPER LEVEL WIND AND RAINFALL VARIABILITY}

\subsection{Interannual variability of upper level winds over South America}

As discussed in the Introduction, Rossby wave trains on the Southern Pacific Ocean generated by ENSO heating anomalies have been linked with rainfall over subtropical SA in spring (Montecinos et al. 2000; Mo and Paegle 2001; Paegle et al. 2002). A similar link has been found in fall between upper level wind circulation and precipitation, but this particular one seems not related to ENSO (Cazes-Boezio et al. 2003). To investigate the relationship between precipitation over SESA and upper level wind anomalies, we described the winds interannual variability by the mean of an EOF analysis. 
The EOF analysis was performed on winds over SA (10N, 60S; 90W, 30W) at the $200 \mathrm{hPa}$ level, for the period 1948-2002, for ON, JF, AM and JA individually. As a first step, we computed seasonal anomalous meridional and zonal wind fields with respect to the long-term mean of each season at each grid box. Next, we merged the two components in one vector for each time step, and obtained eigenvectors and eigenvalues of the covariance matrix.

Depending on season, the variability of upper level winds over SA is dominated by 2-3 distinct modes, which together account for 40 to $55 \%$ of the total variance; for all four seasons the eigenvalues spectrum levels off after those 2-3 leading modes, and higher order modes are indistinguishable. The patterns associated with the $1^{\text {st }}$ leading mode in $\mathrm{ON}$ and $\mathrm{AM}$ and with the $2^{\text {nd }}$ leading mode in JF and JA have very similar structure, and account for a large fraction of the total variance in each season: $25 \%$ in $\mathrm{ON}, 19 \%$ in JF, $28 \%$ in AM and $14 \%$ in JA. They consist of a continental scale vortex, centered on the Atlantic coast of subtropical SA at around 25S; 50W (see as an example in Fig.2). For all four seasons results are robust: performing the EOF analysis by considering the period 1959-2001 does not affect the outcomes. Regression maps of corresponding PC on upper level winds over the South Pacific Ocean resemble the PSA1 pattern in spring and fall, while in summer the pattern is barely identifiable (not shown). The vortex has a quasi-barotropic structure in spring, summer and fall, as shown for example in AM, by the composite of wind at 200, 500 and $850 \mathrm{hPa}$ over events in which the normalized $\mathrm{PC}$ of upper level winds variability is greater than 1 (see Fig.3). Similar patterns are found for the other seasons, and composites over values of the PC smaller than -1 (not shown) depict the same circulation except for the opposite sign. 
We find a significant positive simultaneous correlation, around 0.6 , between the corresponding PCs of these modes and bi-monthly mean precipitation anomaly over SESA in spring, summer and fall. In winter the relationship does not hold and we no longer discuss this season. For simplicity, we consider the same region (SESA) for all seasons, even thought slight differences in the location of larger precipitation anomalies appear in $\mathrm{ON}$, while the location is largely comparable in JF and AM (Figs. 4, 5, 6). None of the other PCs is significantly correlated with precipitation in any season.

We have repeated the EOF analysis with the ERA40 dataset and obtained patterns match very closely with those obtained using the NCEP-NCAR reanalysis. There are small differences, of the order of $1-2 \%$, in the amount of variance accounted by each mode. The correlation between corresponding PCs is 0.95 in $\mathrm{ON}, 0.75$ in JF and 0.93 in AM. Finally, the correlations between ERA40 PCs and mean precipitation over SESA are basically the same in ON and AM while in JF the correlation is 0.45 instead of the 0.67 we reported (using on the NCEP data). For this reason, the results regarding the JF season are less reliable.

\subsection{The relationship between rainfall and winds}

In all seasons, intense trade winds entering northern SA are deflected southward over the western tropical regions forming the SALLJ (Fig. 1). Besides this common behavior, the circulation has a significant seasonal variability. In $\mathrm{ON}$ moisture flux is intense down to $25 \mathrm{~S}$ where it weakens and becomes southeastward over SESA. In JF the overall circulation is at its maximum strength 
and over the eastern Brazil (around 20S-50W) it merges with the moisture flux coming from the Atlantic subtropical high. In AM the SALLJ circulation is weakest but nevertheless it reaches SESA at $65-70 \mathrm{~W}$ where it is oriented southeastward; its main source is the easterlies between 515S. A comparison of the precipitation patterns in Fig.1, reveals an interesting feature over SESA: precipitation amounts are fairly constant throughout the seasons (Diaz et al. 1998). This is particularly evident in the fall season when the dominant pattern of precipitation has retreated to the north and precipitation amounts over SESA contrast with those of the surrounding areas. Different phenomena are responsible for precipitation in different seasons while the SALLJ is a common element in providing moisture to the region. Indeed, larger precipitation amounts over SESA closely correspond to the SALLJ pattern. To explore the mechanisms underlying the relation between upper level winds variability and precipitation anomalies over SESA, we examine in detail the composites of rainfall and vertically integrated moisture flux anomalies over events in which the normalized PC is greater (smaller) than 1 (-1). All composites are, at least partially, $90 \%$ significant according to the Montecarlo test. We also evaluate other water budget terms by simple regression of the $\mathrm{PC}$ onto the latent heat flux (evaporation) and the divergence of the vertically integrated moisture fluxes. Positive (negative) events occurred in ON of 1972, 1982, 1984, 1994 and 1997 (1962, 1964, 1970, 1971, 1974, 1976, 1983, 1989, 1998 and 1999), in JF of 1971, 1973, 1984 (1962, 1979, 1980, 1982 and 1991), in AM of 1959, 1973, 1980 and $1984(1960,1962,1968,1978,1979$ and 1982).

In $\mathrm{ON}$, in the composite of positive events, the anomalous moisture flux is characterized by a southward extended SALLJ; precipitation is increased over SESA where the moisture flux 
associated with the SALLJ is diverted to the east (Fig.4a). The circulation at higher levels (not shown) conveys moisture to the south west from the tropical Atlantic Ocean at about 12S, which results as a secondary source of moisture for SESA. Negative precipitation anomalies are observed over the SACZ creating the dipole in precipitation previously reported by several authors (Fig.4a). In the composite of negative events (Fig.4b) the dipole in precipitation reverses sign, even though there are slight differences in the intensity and structure of the two centers and, particularly over SESA precipitation anomalies extend further southward (around 38S). The moisture flux distribution is characterized by a well defined cyclone centered over the ocean at $27 \mathrm{~S}-35 \mathrm{~W}$, which represents a marked asymmetry with respect to the positive phase of the circulation. The associated moisture flux anomalies weaken and confine the SALLJ to the north, consistently with reduced precipitation over SESA. Southwesterly anomalies over eastern Brazil confine the moisture flux from the tropical Atlantic over the SACZ and contribute in increasing precipitation there. The circulation found in the negative phase contrasts with the findings by Díaz and Aceituno (2003) who interpreted the positive precipitation anomalies over the SACZ as the result of a diverted SALLJ into that region, while here we suggest that increased precipitation over the SACZ is the result of the confined moisture flux from tropical western Atlantic Ocean over the area. We notice over Chile the presence of precipitation anomalies of the same sign and intensity as those over SESA for both phases of the circulation.

The intensity and location of precipitation anomalies are in qualitative agreement with the divergence of vertically integrated moisture flux pattern (Fig.4c): the regression map shows convergence over SESA and divergence over the SACZ corresponding to the anticyclonic phase 
of the vortex (positive PC values) and vice versa. In particular precipitation anomalies in the cyclonic phase (Fig.4b) are consistent with convergence over the region receiving direct moisture from the ocean (SACZ) and divergence over SESA. Evaporation anomalies are not an important contributor to the water budget since their magnitude is about one order of magnitude smaller than precipitation anomalies (Fig.4c). We disregard the large anomalies over the Andes between $80-70 \mathrm{~W} ; 0-30 \mathrm{~S}$ since we believe them to be artifacts of the reanalysis data. Asymmetries among positive and negative cases and the limited accuracy of reanalysis data to capture small differences in the moisture flux do not allow for drawing firm quantitative conclusions. Nevertheless, the results presented support the hypothesis that precipitation anomalies are created by the circulation anomalies we documented.

In JF a well defined vortex centered on the eastern coast of SA characterizes both composites. In the positive phase, corresponding to the southwestern - SESA (northeastern - SACZ) branch of the vortex, positive (negative) precipitation anomalies are observed (Fig.5a). Anomalies of the opposite sign are observed in the negative phase of the circulation. The upper level circulation has been described as a stationary Rossby wave for JFM by Robertson and Mechoso (2000), who also reported a dipole in the vertical velocity consistent with precipitation anomalies.

In the anticyclonic (positive) circulation (Fig. 5a) the main source of moisture for SESA is the SALLJ. To better identify this feature we also show the composite of the total vertically integrated moisture flux for the positive phase of the circulation (Fig.5c). The anomalous vortex depicted in Fig.5a is consistent with a strengthened and zonally broader SALLJ. This results in large anomalous moisture flux that reaches more meridional $(\sim 35 \mathrm{~S})$ and western $(\sim 65 \mathrm{~W})$ regions. 
As for ON, a secondary source of moisture originates in the tropical western Atlantic, it merges with the eastern flank of the SALLJ over central Brazil (Fig.5c) and reaches SESA. The pole of intense precipitation over Ecuador, Peru and Colombia corresponds to a locally reduced intensity of the flux from the trade winds (Fig.5a). In the opposite phase (Fig.5b) the SALLJ is diverted eastward and, together with the northerly moisture flux from equatorial Atlantic at 50W, it creates positive precipitation anomalies over the SACZ and central Brazil, and negative anomalies over SESA. As for ON, precipitation anomalies of the same sign as in SESA are observed over Chile; however, compared to those in ON, they have a smaller extension.

The regression map of the divergence, is in qualitative agreement with precipitation anomalies: it shows a narrow area of negative anomalies over the SACZ and a positive area to the south of SESA. Clearly the correspondence of the divergence pattern with those of precipitation is not strict. An exact correspondence between the anomalous moisture flux and precipitation patterns is not expected in view of the asymmetries between the positive and negative cases, together with the already discussed lack of accuracy of the reanalysis data. As for ON, in JF evaporation anomalies are not an important contributor to the water budget (not shown).

In $\mathrm{AM}$ in the anticyclonic phase the vortex is centered over the ocean but the circulation extends over the central-eastern part of the continent combining the SALLJ and moisture from the western tropical Atlantic. Consistently, positive precipitation anomalies are observed over SESA (Fig.6a). On the contrary, the circulation confines the SALLJ to the north in the cyclonic phase of the circulation and negative precipitation anomalies are found in SESA (Fig.6b). In both cases, a dipole of precipitation is observed over the north-northeast part of the continent possibly 
due to the combined effects of the eddy and the trade winds from the Equatorial Atlantic. As for the previous seasons, precipitation anomalies are observed over Chile and have, in this season, the largest spatial extension. The regression over the divergence field presents a broad area of positive anomalies and it is in qualitative agreement with precipitation anomalies (Fig.6d). Similarly to the other seasons, evaporation anomalies contribute little to the water budget (Fig.6c).

Summarizing, we reported asymmetries between the cyclonic and anticyclonic phase of the circulation which affect precipitation anomalies over SESA and the SACZ. Precipitation anomalies are not only the result of a deflected SALLJ over the region presenting positive precipitation anomalies (Diaz and Aceituno 2003; Liebmann et al.2004; ), but they are the result of the entire trade winds moisture flux transport. We emphasize that the source of moisture impacting precipitation in different seasons can be different, which is a result not previously reported in the literature. We also indicate other centers of precipitation anomalies over SA modulated by interannual wind variability, which are particularly consistent over Chile.

\subsection{On the relationship between the Atlantic Ocean and precipitation over SESA in the fall season}

In the fall season, some authors (Diaz et al. 1998 and Cazes-Boezio et al. 2003) have suggested an impact of subtropical Atlantic SSTs in the region close to the South American coast on precipitation over SESA. Instead, our analysis suggests that the wind anomalies induce SST 
anomalies, which confirms the relationships reported but give them a different interpretation. In figure 7 we show that corresponding to the anticyclonic phase of the circulation, reduced evaporation (negative latent heat flux anomalies) and consistent increased in the SSTs are observed around 25-30S on the South American coast. If the atmosphere were driven by the ocean, low-level cyclonic and baroclinic circulation would correspond to warmer SSTs. Similar anomalies are found in the opposite case, although negative SSTs anomalies are displaced to the north east, and possibly due to not negligible ocean dynamics. In this context, our results extend for the fall season the concept of the atmosphere-forcing-the-ocean under the SACZ in summer as suggested by Robertson and Mechoso (2000) and supported by Chaves and Nobre (2004).

\section{LONG RANGE FORECASTS}

\subsection{Empirical model}

Motivated by the relationship between upper level wind variability and precipitation anomalies reported above, we developed a methodology to predict precipitation over SESA $(60-45 \mathrm{~W} ; 24$

- $38 \mathrm{~S}$ ). Our methodology is the same in all seasons, except that the mode used as predictor depends on the season. This dependence is discussed in section 3.1.

To associate an uncertainty to the prediction, the forecast is provided in the form of a distribution each year. We assume a normal distribution since the observed mean precipitation over SESA is approximately Gaussian in spring, summer and fall during 1959-2001. In the initial development 
of the methodology we have been following the idea Coelho et al. (2004) developed for the prior distribution of Niño 3.4 SST.

The linear regression between the predictor and predictant is determined in cross-validation, leaving out the data of the year being forecasted. The computation of the linear regression is therefore repeated for each year. This technique allows the maximum use of the dataset to develop the model and verify it without including any piece of information already considered.

For a particular year, the mean of the empirical distribution is obtained from the equation of the regression line and the value of the predictor for that year; the standard deviation is the root mean square of residuals of the regression line $\left(\sigma_{\mathrm{o}}\right)$ increased by two terms which account for the finite size of the dataset and the distance of the predictor from its time mean (Wilks, 1995). In compact notation:

$$
\begin{aligned}
& P\left(\theta_{t} \mid \psi_{t}\right) \approx N\left(\mu_{o t}, \sigma_{o t}^{2}\right) \\
& \mu_{o t}=\beta_{o}+\beta_{1} \psi_{t} \\
& \sigma_{o t}=\sigma_{o}\left[1+\frac{1}{n-1}+\frac{\left(\psi_{t}-\overline{\psi_{t}}\right)^{2}}{\sum_{i \neq t}^{n-1}\left(\psi_{i}-\overline{\psi_{t}}\right)^{2}}\right]^{1 / 2}
\end{aligned}
$$

where $\mathrm{P}\left(\theta_{\mathrm{t}} \mid \psi_{\mathrm{t}}\right)$ stands for probability distribution of the variable $\theta_{\mathrm{t}}$ given $\psi_{\mathrm{t}}$, and the subscript $\mathrm{t}$ represents the year the forecast refers to. In our case $\theta_{\mathrm{t}}$ is precipitation and $\psi_{\mathrm{t}}$ is the PC selected 
as predictor. $\mathrm{N}\left(\mu_{\mathrm{ot}}, \sigma_{\mathrm{ot}}\right)$ stands for Gaussian distribution with mean $\mu_{\mathrm{ot}}$ and standard deviation $\sigma_{\mathrm{ot}}$; $\sigma_{\mathrm{o}}$ is the root mean squared error of the regression line, $\mathrm{n}$ is the number of years used to compute the linear regression, $\bar{\psi}_{t}$ is the time mean of the predictor over all years except the $\mathrm{t}^{\text {th }}, \beta_{0}$ and $\beta_{1}$ are the coefficients of the regression line.

Since the highest correlation between precipitation and $\mathrm{PC}$ is found in the case with no time lag between corresponding timeseries, we examine the potential skill of the method by using PCs computed from the reanalysis. To test the method in a more realistic forecast framework the PC must be predicted as well. The PC forecast is defined by the projection of wind anomalies of the DEMETER hindcasts at $200 \mathrm{hPa}$ onto the EOF patterns computed with NCEP reanalysis. An alternative method is to perform the EOF analysis directly on the GCMs fields. We chose the first method since we consider EOF modes as known features of the system, available each time a forecast is released, and we can reasonably expect the variability of the forecasted wind fields to be smaller than in the observation. For a simple evaluation of our results we compute the skill score as the mean squared error with respect to climatology (Wilks, 1995); this procedure is commonly used in forecast verification. Through the values of the variance, we provide a confidence estimate on the results.

\subsection{Potential predictability}

The empirical method provides useful predictions of precipitation anomalies when PCs based on reanalysis data are used as predictors. The skill score is about 0.4 in spring, summer and fall (TABLE 1). The value is higher than the one of the bias corrected EMU as well as the one 
reported by IRI (Goddard et al. 2003), and also by COF (Berri et al. 2005). Although the variability reproduced by the method is still smaller than the observed one (Fig.8), the empirical model can forecast a large range of values. Corresponding to large anomalies, there is, in most cases, an $80 \%$ probability of correctly forecasting values below or above the climatology (see for e.g. 1982 and 1997 in ON, 1984 in JF, 1982 in AM in Fig.8). Further, the method is reliable in general and only few values fall outside of the $80 \%$ confidence interval. As reference for the reader, the mean ${ }^{1}$ standard deviation of the forecasts is $0.94,0.77,0.96 \mathrm{~mm} /$ day for $\mathrm{ON}$, JF and AM respectively.

\subsection{Experimental forecasts}

Results based on using PCs obtained by reanalysis data indicate the potential of the $200 \mathrm{hPa}$ wind field as predictor and encouraged us to test our method in a more realistic forecast framework. As expected, the model' skill is lower when forecasted PCs are used as predictor. Nevertheless, skillful predictions are still obtained in spring (TABLE 1, and Fig.9). The skill score in summer and fall drops to zero from 0.4 of the potential predictability case. In fall and summer the method is not reliable since at the $80 \%$ confidence level it would provide the same forecast every year and more than $20 \%$ of the forecasts fall outside this interval. We note that variability of the predictions is very small relative to that of the observations (as an e.g., see Fig.9).

\footnotetext{
${ }^{1}$ There is a value for the standard deviation for each forecast being the distribution different each year.
} 
We next explore why real predictions are skilful in spring only and the reasons for the not realized predictability of fall and summer. It is apparent that the only cause for the decrease in skill is the use of forecasted wind fields. In this regard, the correlation between forecasted and PCs based on the reanalysis is 0.6 in spring but it is not significant in summer and fall. The wind field climatology is well simulated by EMU in all seasons (not shown), but the anomalous circulation is only reasonably captured in spring. Composites of NCEP reanalysis fields over events in which the normalized PC1 is greater (smaller) than $1(-1)$ closely resembles the EOF pattern in spring, summer and fall: there is a vast anticyclonic (cyclonic) circulation centered over the eastern coast of subtropical SA reaching the north western coast of the continent. A pattern resembling the eastern part of the PSA 1 mode appears to the south west of the domain in the composite of $\mathrm{ON}$ and $\mathrm{AM}$. The corresponding composites for spring from EMU do not reproduce the anomalies in the Tropics. Nevertheless, a signature of the PSA pattern over the south west and the vortex are captured, although its center is slightly displaced to the east (Fig.10). In summer the eddy is not found in either of the composites (not shown). In fall, there is a weak signature of the PSA pattern, and the eddy is captured but its center is displaced about 20 degrees in the composite of positive events, while in the composite of negative events the whole pattern is missed (Fig.11).

When the EOF pattern is projected onto an anomalous field not well captured in the forecasts, the resulting value of the "forecasted PC" is small. Thus the relationship between precipitation and PC found by using observational data cannot be reproduced. 
These results are consistent with ENSO triggering a PSA1-like regime through Rossby wave propagation in spring only (Cazes-Boezio et al. 2003). Robertson and Mechoso (2003) proposed a different interpretation of the PSA-like regimes as geographically fixed circulations in fall, winter and, to a lesser extent in spring. Nevertheless, they also found a strong correlation between ENSO and the frequency of occurrence of the PSA-like regimes in spring. We can reasonably summarize that in southern spring ENSO, one of the strongest forcing of the climate system in the interannual timescale, is associated to, and even possibly generates, the anomalous vortex over SA. This is consistent with a higher capability of current GCMs in reproducing the mechanism and pattern in the wind field. We have conducted a preliminary investigation on the relationship between the vortex over SA, the PSA and ENSO for the three seasons and we found that ENSO may trigger the leading PSA, which in turn is related to the vortex over SA. However this chain of elements presents differences in different seasons and does not fully account for the occurrence of the vortex over SA (Zamboni 2009). At present for the seasons other than spring, the mechanisms responsible for the occurrence of the vortex over SA are unknown and further analysis is required to understand why the atmosphere over SA presents the described preferred configuration and thus properly represent it in the GCMs.

\subsection{Predictability associated with ENSO}

Corresponding to stronger ENSO events we can expect predictability to increase because of a larger the 'signal-to-noise ratio'. In our study, this is of particular interest for the spring season since we may expect to find the circulation anomalies we have discussed at a more frequent rate, 
and the GCMs to better capture them. To investigate whether the predictability of the system and the skill of the forecast are accordingly higher, we developed and tested the empirical model considering ENSO events only for the spring season. For this test, we adopted the definition of ENSO events by the Japan Meteorological Agency (JMA), which classifies years as events if the 5 months running mean of SSTs anomalies over $4^{\circ} \mathrm{S}-4^{\circ} \mathrm{N}, 150^{\circ} \mathrm{W}-90^{\circ} \mathrm{W}$ is equal or higher ( lower ) than $0.5^{\circ} \mathrm{C}\left(-0.5^{\circ} \mathrm{C}\right)$ for 6 consecutive months. According to this definition there are 10 El Niño and 10 La Niña from 1959 to 2001.

The skill score of precipitation forecasts in spring is doubled (0.46) using forecasted PC of wind as predictor corresponding to the occurrence of ENSO events. To evaluate the effects on the skill of the strong events 1982/83 and a 1987/88, we applied the method after having removed those cases. The skill score results 0.5 .

To use our method in a real forecast framework a prediction for the occurrence of an ENSO event is required. We do not believe this requirement to make a significant difference since we expect state-of-the-art GCMs to be reasonably sensitive to ENSO events. For example, the correlation between the observations and EMU over $4^{\circ} \mathrm{S}-4^{\circ} \mathrm{N}, 150^{\circ} \mathrm{W}-90^{\circ} \mathrm{W}$ is 0.94 . The increase of the skill when the empirical model is applied to ENSO events only reveals a further potential of the method besides the assumed linearity of the response of the system to El Niño and La Niña, and the relationship between precipitation and PC of upper level wind over SA.

\section{CONCLUSIONS}


We have shown evidence of a simultaneous linear relationship at interannual timescales between precipitation anomalies over SESA (60-45W; 24-38S), a region that comprises parts of La Plata River basin in SA, and the interannual variability of upper level wind over SA. The relationship holds in spring, summer and fall. The anomalous wind pattern related to precipitation anomalies is very similar in all seasons: it consists of a continental scale vortex, centered on the Atlantic coast of subtropical SA at $25 \mathrm{~S} ; 50 \mathrm{~W}$ (Fig. 2). This configuration is the $1^{\text {st }}$ leading EOF mode in ON and AM and the $2^{\text {nd }}$ leading EOF mode in JF; they account for $25,19 \%$, and $28 \%$ of the total variance respectively. In addition, regression maps suggest that the pattern could be related to the PSA1 in spring and fall, while their connection is less evident in summer.

The vortex has a quasi-barotropic structure in spring, summer and fall (see as an example Fig.3). The circulation associated with the anticyclonic phase of the upper level vortex combines at lower-levels moisture fluxes from the SALLJ and the western tropical Atlantic contributing to increased precipitation over SESA in spring, summer and fall, and diminished precipitation over the SACZ in spring-summer thus creating the north-south precipitation dipole previously reported by several authors. During the cyclonic phase of such vortex, when increased precipitation is observed over the SACZ (in spring-summer) and decreased precipitation is observed over SESA (in spring, summer and fall), seasonal differences arise. In spring precipitation anomalies are associated with a confined moisture flux from the tropical Atlantic over the SACZ and with a weakened SALLJ, in summer they are associated with an eastward diverted SALLJ toward the SACZ, while in fall the SALLJ is weakened and confined to the north. 
We also indicate other centers of precipitation anomalies over SA modulated by interannual wind variability; particularly over Chile precipitation anomalies are consistent throughout the seasons and of the same sign of those over SESA.

Even though asymmetries among positive and negative cases and the limited accuracy of reanalysis data to detect small differences in the moisture flux do not allow for drawing firm quantitative conclusions, our results do support the hypothesis that precipitation anomalies are associated with the quasi-barotropic circulation we identified. In fact, the intensity and distribution of precipitation anomalies are in qualitative agreement with those of vertically integrated moisture flux divergence, while evaporation anomalies are one order of magnitude smaller (see Figs. 4, 5, and 6).

In the fall season, some authors (Diaz et al. 1998 and Cazes-Boezio et al. 2003) have suggested that subtropical Atlantic SST anomalies near the South American coast are linked to precipitation over SESA. We suggest that wind circulation anomalies induce the one in the SSTs (see Fig.7). Since oceanic anomalies are forced by atmospheric anomalies, the former are associated with, but cannot be used as predictor of the latter.

We have shown that long-range seasonal precipitation forecasts over SESA from operational centers have low skills (Goddard et al. 2003; Berri et al. 2005; Coelho et al. 2006). In view of this, we proposed an empirical model as alternative method to provide rainfall predictions in spring, summer and fall based on the relationship we documented between upper level wind variability and precipitation. 
In order to provide an estimate of the precipitation predictability associated with upper level winds in a real forecast, we carried out the analysis using predictors obtained by the reanalysis data first and forecasted predictor values next. For the latter investigation, we made use of the DEMETER forecasted 200hPa wind fields.

PCs can potentially be used as predictor of the mean precipitation over SESA in spring, summer and fall: the skill score is about 0.4 in all cases. The empirical model can forecast a large range of values, and it is reliable with just few values falling outside the $80 \%$ confidence interval (see Fig.8).

In summer and fall the DEMETER GCMs forecasts of wind anomalies limit the method's potential: the forecasted wind anomalies are not well captured and consequently the relationship between precipitation and PC found in the observations is not well reproduced either (see Figs. 10 and 11). Wind anomalies are reasonably well captured in spring, and skilful precipitation predictions are obtained in this season, the skill score being 0.26 .

It is furthermore shown that predictions for spring are improved when the method is applied in the occurrence of ENSO events; in this case the skill score is 0.46 . The result is consistent with the hypothesis that ENSO is able to trigger a pattern associated with the PSA1 in spring (CazesBoezio et al. 2003; Robertson and Mechoso 2003). For the seasons other than spring, the mechanisms responsible for the occurrence of the vortex over SA are unknown and further analysis is required to understand why the atmosphere over SA presents the described preferred configuration and thus properly represent it in the GCMs. 
Acknowledgments: This research was supported by NOAA under the grant NA05OAR4310009 and by the Dipartimento di Matematica, Universitá degli Studi di Trieste, Italy. The authors wish to thank Dr. G. Cazes-Boezio and Dr. A.W. Robertson for the stimulating discussions and helpful suggestions.

\section{References}

Berbery, E.H., and E.A. Collini, 2000: Springtime Precipitation and Water Vapor Flux over Southeastern South America. Mon. Wea. Rev.,128, 1328-1346.

Berri, G.J, P.L. Antico, L. Goddard, 2005. Evaluation if the Climate Outlook Forum's Seasonal Precipitation Forecasts of Southeast South America During 1998-2002. International J. of Climatology, 25:365-377. 
Casarin, D. P., and V. E. Kousky, 1986: Anomalias de precipitação no sul do Brasil e variações na circulação atmosférica. Rev. Bras. Met., 1, 83-90.

Cazes-Boezio, G., A.W. Robertson and C.R. Mechoso, 2003: Seasonal Dependence of ENSO teleconnections over South America and Relationships with Precipitation over Uruguay. $J$. Climate, 16:1159-1176.

Chaves, R. R., and P. Nobre (2004), Interactions between sea surface temperature over the South Atlantic Ocean and the South Atlantic Convergence Zone, Geophys. Res. Lett., 31, L03204, doi:10.1029/2003GL018647.

Coelho, C.A.S., S. Pezzulli, M. Balmaseda, F.J. Doblas-Reyes, and D.B. Stephenson, 2004: Forecast Calibration and Combination: A Simple Bayesian Approach for ENSO. J. Climate, 17, 1504-1516.

Coelho, C.A.S., D.B. Stephenson, M. Balmaseda, F.J. Doblas-Reyes, and G.J. van Oldenborgh, 2006: Toward an Integrated Seasonal Forecasting System for South America. J. Climate, 19, 3704-3721.

Diaz, A.F., C.D. Studzinski, and C.R. Mechoso, 1998: Relationships between Precipitation Anomalies in Uruguay and Southeastern Brazil and Sea Surface Temperature in the Pacific and Atlantic Oceans. J. Climate, 11:251-271. 
Diaz, A. and P. Aceituno, 2003: Atmospheric Circulation Anomalies during Episodes of Enhanced and Reduced Convective Cloudiness over Uruguay. J. Climate, 16: 3171-3185.

Ferreira, R.N., T.M. Rickenbach, D.L. Herdies, and L.M.V. Carvalho, 2003: Variability of South American Convective Cloud Systems and Tropospheric Circulation during January-March 1998 and 1999. Mon. Wea. Rev., 131, 961-973.

Goddard, L., A.D. Barnston, and S.J. Mason，2003: Evaluation of the IRI's "Net Assessment" Seasonal Climate Forecastes 1997-2001. Bulletin of the American Meteorological Society, 12:1761-1781.

Grimm, A.M., V. R. Barros and M. E. Doyle, 2000: Climate Variability in Southern South America Associated with El Niño and La Niña Events. J. Climate, 13:35-58.

Grimm, A.M., 2003: The El- Niño impact on the summer monsoon in Brazil: regional processes versus remote influences. J. Climate, 16: 263-280.

Grimm, A.M., J.S. Pal, and F. Giorgi, 2007: Connection between Spring Conditions and Peak Summer Monsoon Rainfall in South America: Role of Soil Moisture, Surface Temperature, and Topography in Eastern Brazil. J. Climate, 20, 5929-5945. 
Herdies D. L., A. da Silva, M. A. F. Silva Dias, and R. Nieto Ferreira, 2002: Moisture budget of the bimodal pattern of the summer circulation over South America, J. Geophys. Res., 107 (D20), 8075, doi:10.1029/2001JD000997.

Kalnay E., M. Kanamitsu, R. Kistler,W. Collins, D. Deaven, L. Gandin, M. Iredell,S. Saha, G. White, J. Woollen, Y. Zhu, A. Leetmaa, R. Reynolds, M. Chelliah, W. Ebisuzaki, W. Higgins, J. Janowiak, K. C. Mo, C. Ropelewski, J. Wang, R. Jenne and D. Joseph, 1996: The NCEP/NCAR 40-year reanalysis project, Bull. Amer. Meteor. Soc., 77, 437-470.

Liebmann B, Kiladis GN, Vera CS, Saulo AC, Carvalho LMV, 2004: Subseasonal variations of rainfall in South America in the vicinity of the low-level jet east of the Andes and comparison to those in the South Atlantic convergence zone. J. Climate, 17: 3829-3842.

Mo, K.C. and J.N. Paegle, 2001: The Pacific-South American Modes and their Downstream Effects. Int. J. Climatol. , 21:1211-1229.

Montecinos, A., A. Diaz, and P. Aceituno, 2002: Seasonal Diagnostic and Predictability of Rainfall in Subtropical South America Based on Tropical Pacific SST. J. Climate, 13:746-758.

New, M. M. Hulme, and P. Jones, 1999: Representing Twentieth-Century Space-Time Climate Variability. Part I: Development of a 1961-90 Mean Monthly Terrestrial Climatology. J. of Climate 12: 829-856. 
New, M. M. Hulme, and P. Jones, 2000: Representing Twentieth-Century Space-Time Climate Variability. Part II: Development of Monthly Grids of Terrestrial Surface Climate. J. Climate, 13:2217-2238.

Paegle, J.N. and K.C. Mo, 1997: Alternating wet and dry conditions over South America during summer. Mon.Wea.Rev.,126, 3135-3152.

Paegle, J.N. and K.C. Mo, 2002: Linkages between Summer Rainfall Variability over South America and Sea Surface Temperature Anomalies. J. Climate, 15:1389-1407.

Paegle, J.N., C.R. Mechoso, R. Fu, E.H. Berbery, W.C. Chao, T-C. Chen, K.Cook, A. Diaz, D. Enfield, R. Ferreira, A.M. Grimm, V. Kousky, B. Liebmann, J. Marengo, K.C. Mo, J.D. Neelin, J. Paegle, A.W. Robertson, A. Seth, C.S. Vera, and J. Zhou, 2002: Progress in Pan American CLIVART Research: Understanding The South American Monsoon. Meteorologica, 27: 3-32.

Palmer, T. N., 2002. The economic value of ensemble forecasts as a tool for risk assessment: From days to decades. Q. J. R. Meteorol. Soc., 128:747-774.

Robertson, A. W. and C. R. Mechoso, 2000: Interannual and interdecadal variability of the South Atlantic Convergence Zone. Mon. Wea. Rev., 128, 2947-2957. 
Robertson, A. W. and C. R. Mechoso, 2003: Circulation Regimes and Low-Frequency Oscillations in the South Pacific Sector. Mon. Wea. Rev., 131, 1566-1576.

Silva, G. A. M., Ambrizzi, T., and Marengo, J. A.: Observational evidences on the modulation of the South American Low Level Jet east of the Andes according the ENSO variability, Ann. Geophys., $27,645-657,2009$.

Smith, T.M. and R.W. Reynolds, 2004: Improved Extended Reconstruction of SST (1854-1997). J. Climate, 17:2466-2477.

Velasco, I. and J. M. Fritsch, 1987. Mesoscale convective complexes in the America. J. Geophys. Res., 92(D8), 9591-9613.Wilks, D.S., 1995: Statistical Methods in the Atmospheric Sciences. Academic Press, 467 pp.

Zamboni L., 2009: Relationships between Upper Level Circulation over South America and Rainfall over South Eastern South America: A Physical Base for Seasonal Predictions. Ph.D. Thesis. Available at the Universitá degli Studi di Trieste. 

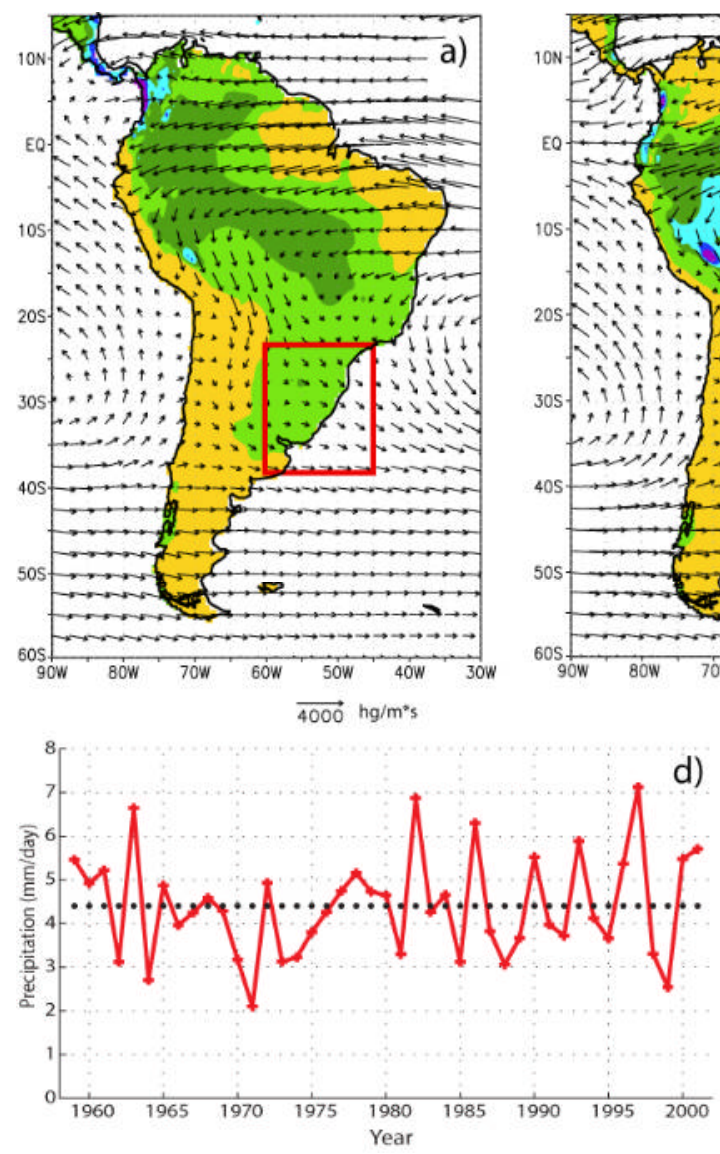
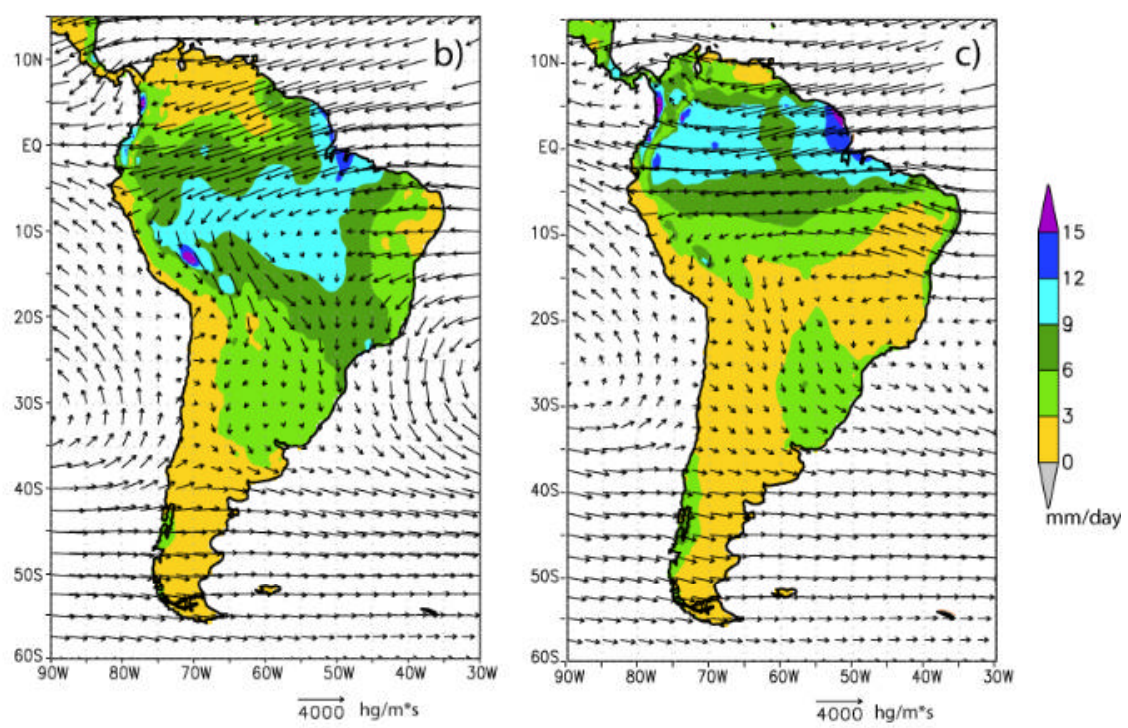

e)

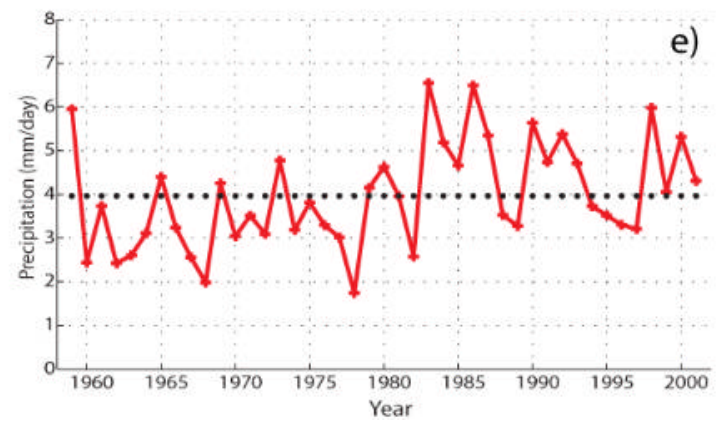

FIG.1 Precipitation (shaded, $\mathrm{mm} /$ day) and vertically integrated moisture flux (vectors, $\mathrm{hg} / \mathrm{m}^{*} \mathrm{~s}$ ) climatology over SA for a) ON, b) JF, c) AM and interannual variability of the mean precipitation over SESA (mm/day) for d) ON and e) AM. The red box in panel a) represents SESA. 


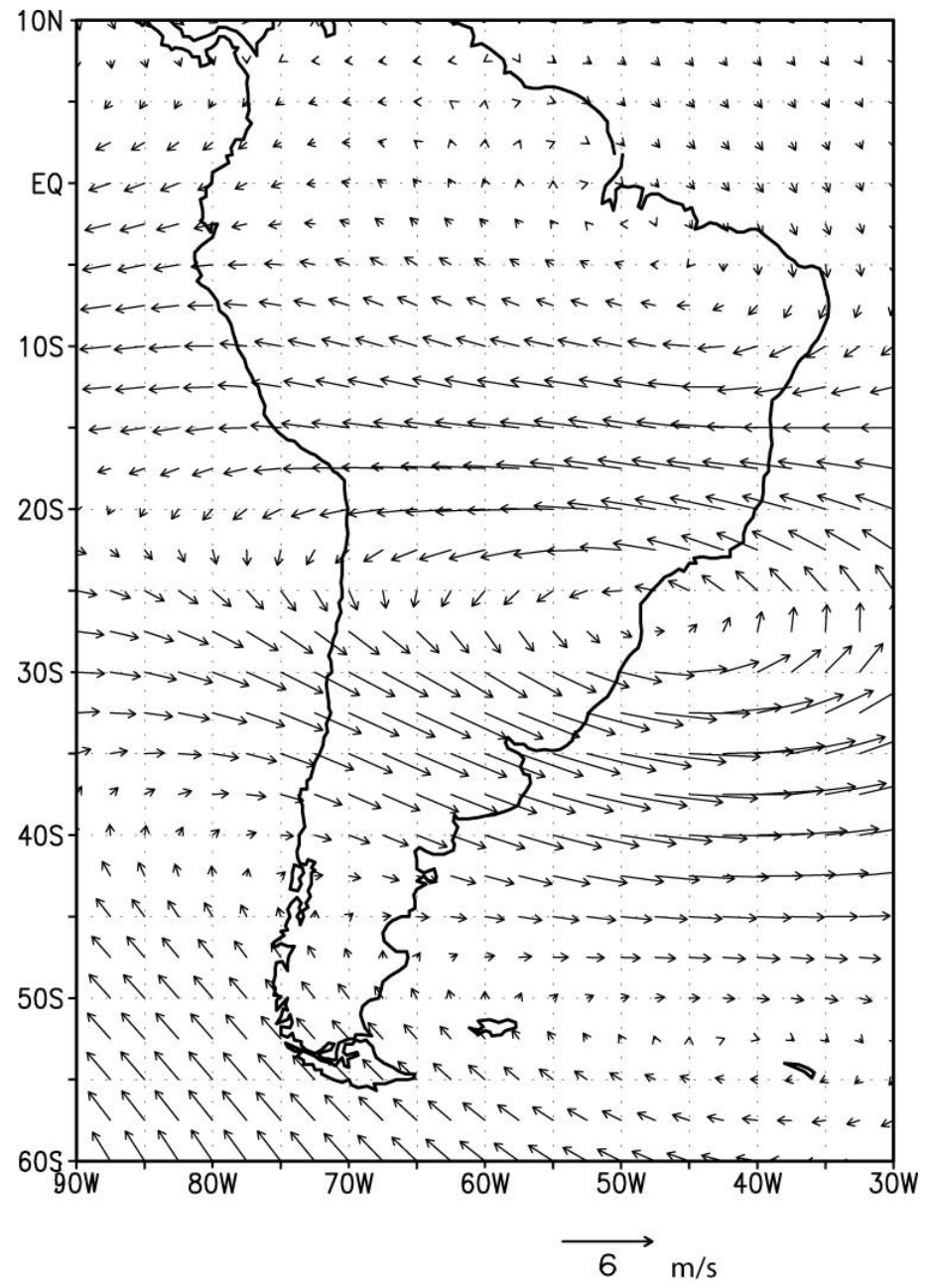

FIG. 2 Leading EOF pattern of 200 hPa interannual variability for ON. 

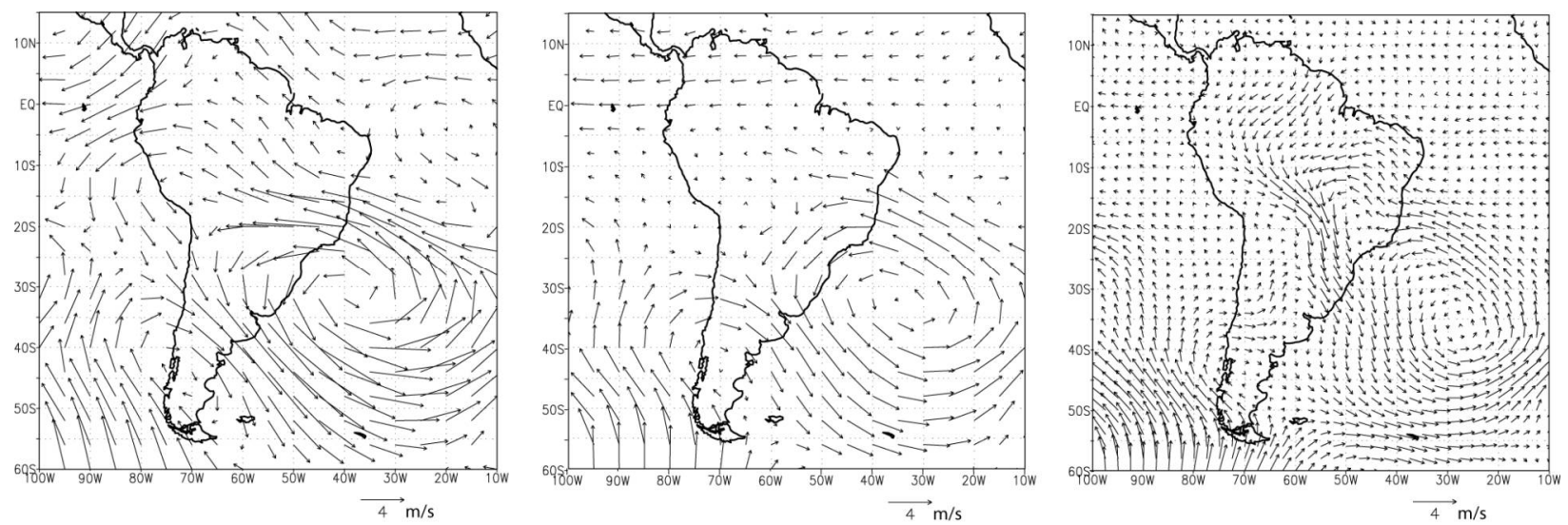

FIG.3 Composite of horizontal wind (m/s) for AM at (from left to right) 200, 500 and 850 hPa over events in which the PC of upper level winds is greater than 1 standard deviation. 

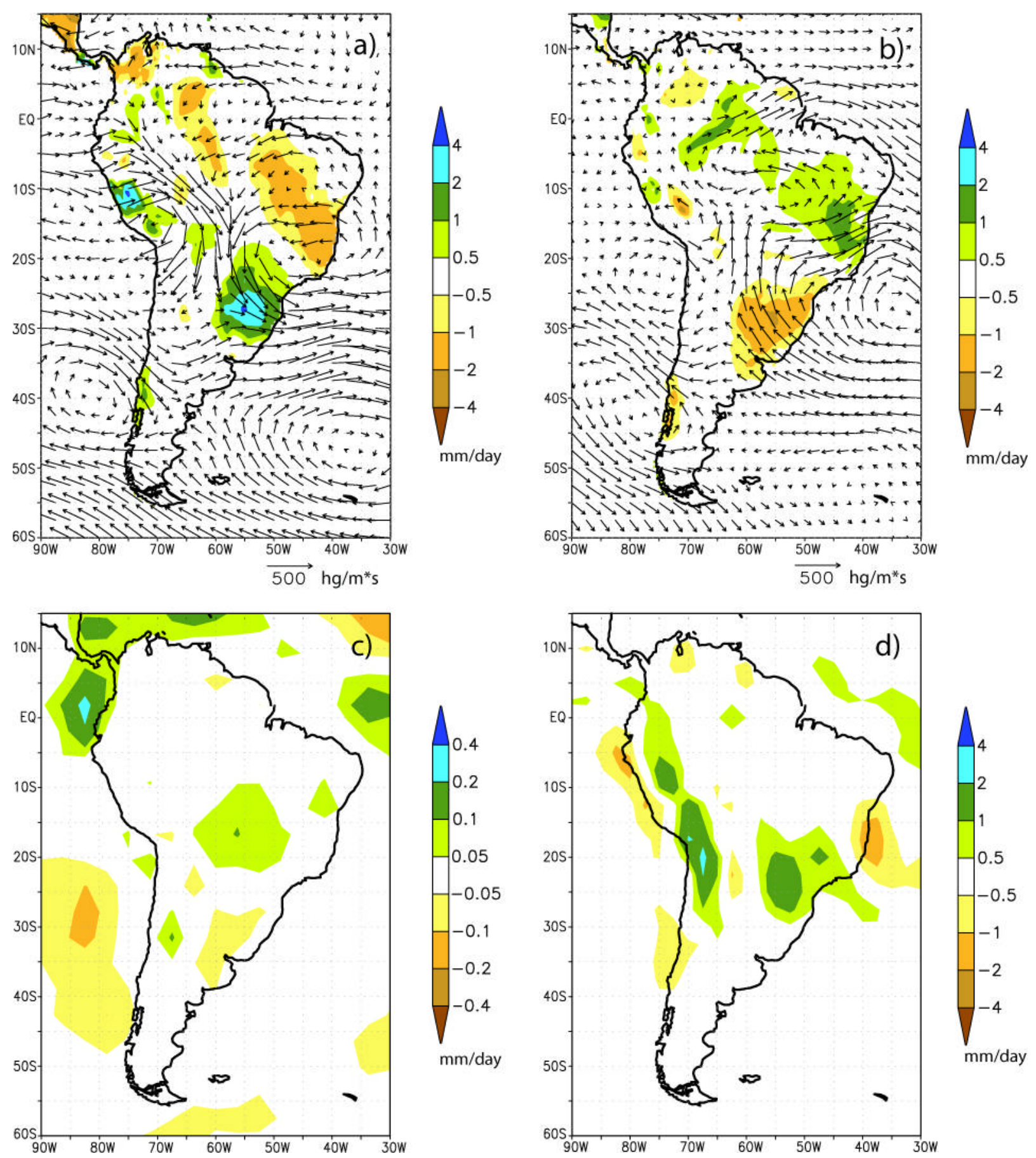

FIG. 4 Water budget terms. Upper panels are the composites of precipitation anomalies (shaded, mm/day) and vertically integrated moisture flux anomalies(vectors, $\mathrm{hg} / \mathrm{m}^{*} \mathrm{~s}$ ) over events in which the PC of $200 \mathrm{hPa}$ winds is a) greater than 1 standard deviation and b) smaller that -1 standard deviation for $\mathrm{ON}$. Bottom panels are the regression of the PC of winds onto c) evaporation (mm/day) and d) divergence of the vertically integrated moisture flux (mm/day). Note the scale of the evaporation represents anomalies one order of magnitude smaller compared to the other fields. 

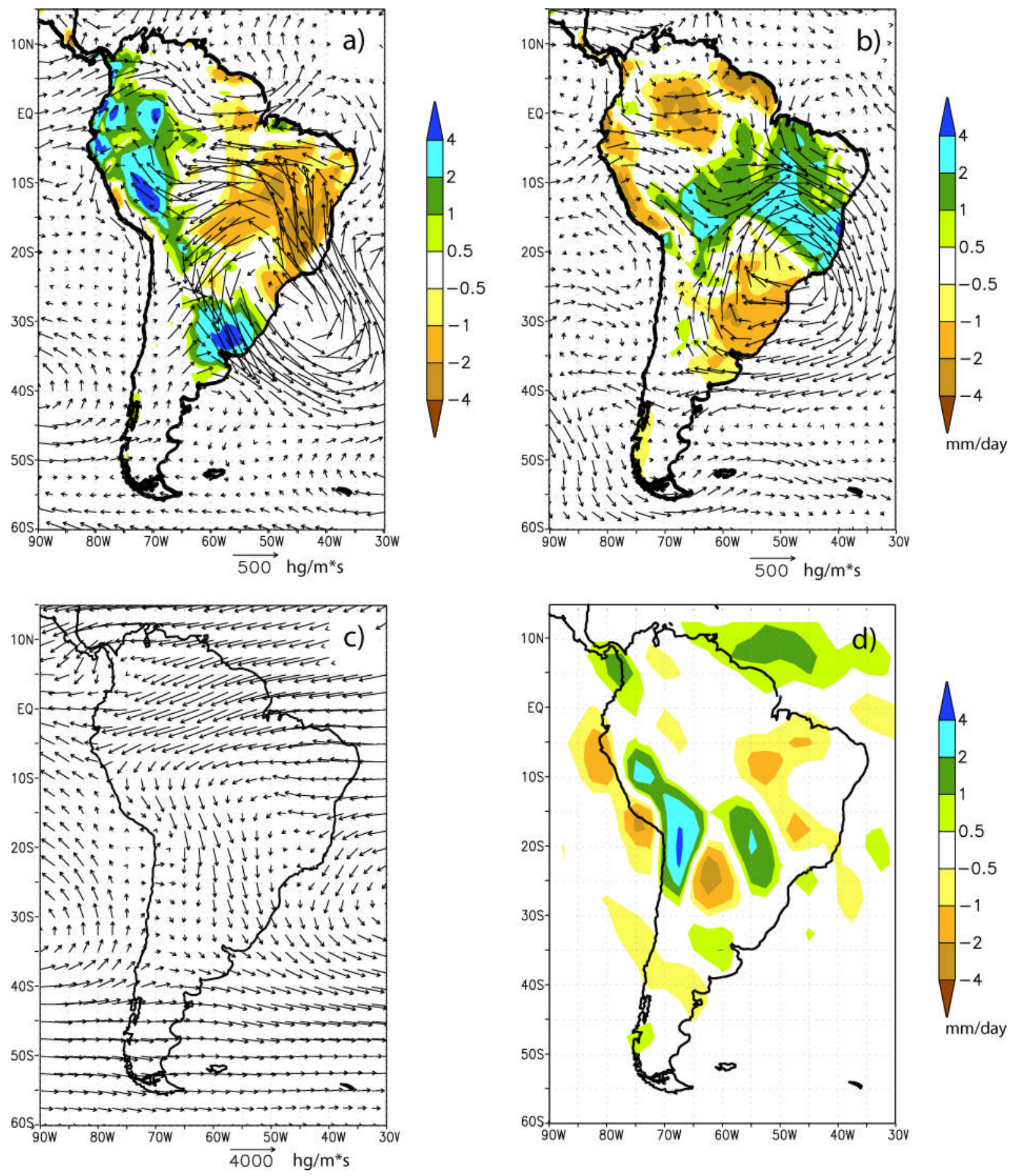

FIG. 5 As in FIG. 4 but for JF and C) is the composite of the total vertically integrated moisture flux over events in which the PC of $200 \mathrm{hPa}$ winds is greater than 1 standard deviation. 

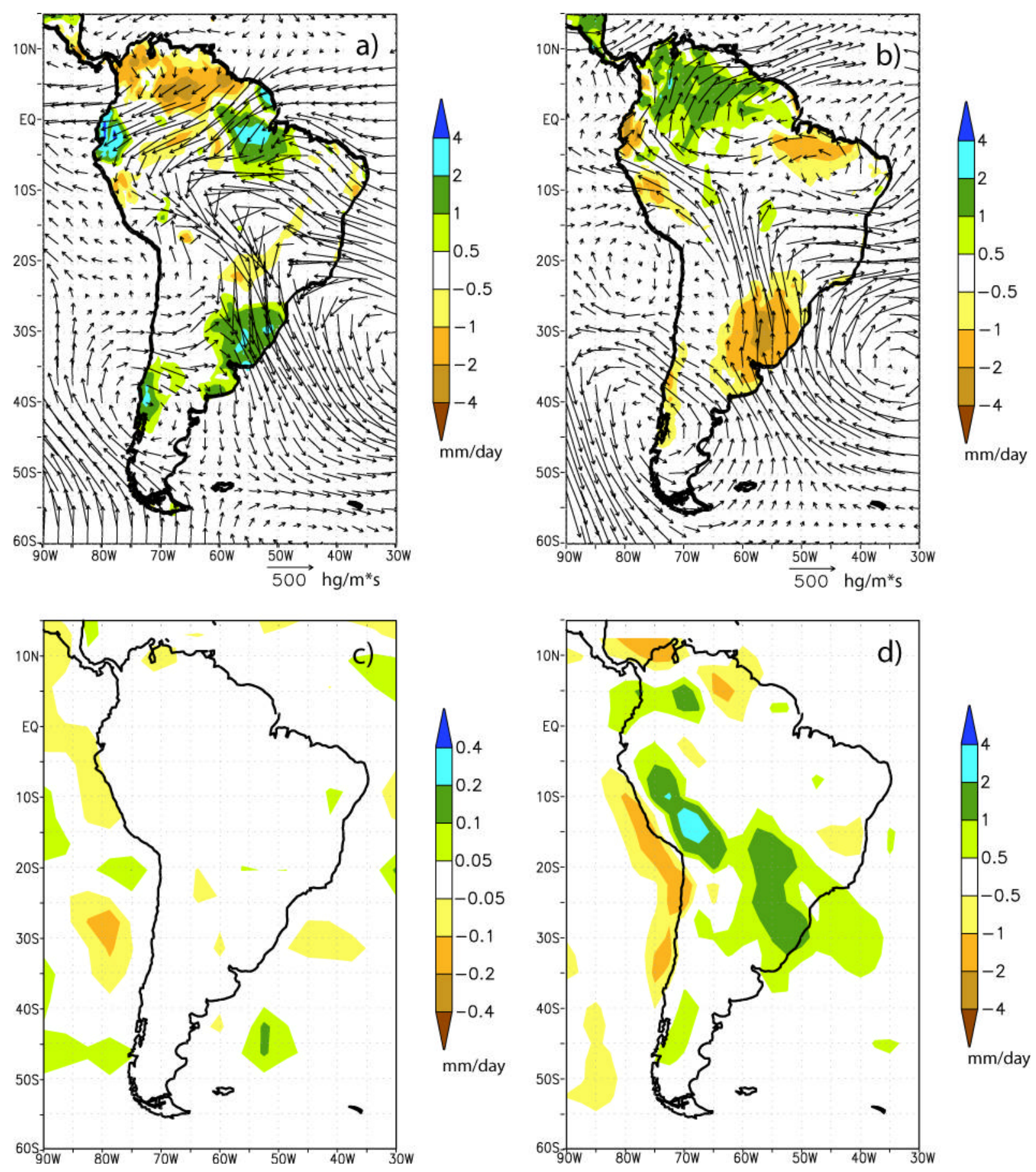

FIG. 6 As in FIG.4 but for AM. 

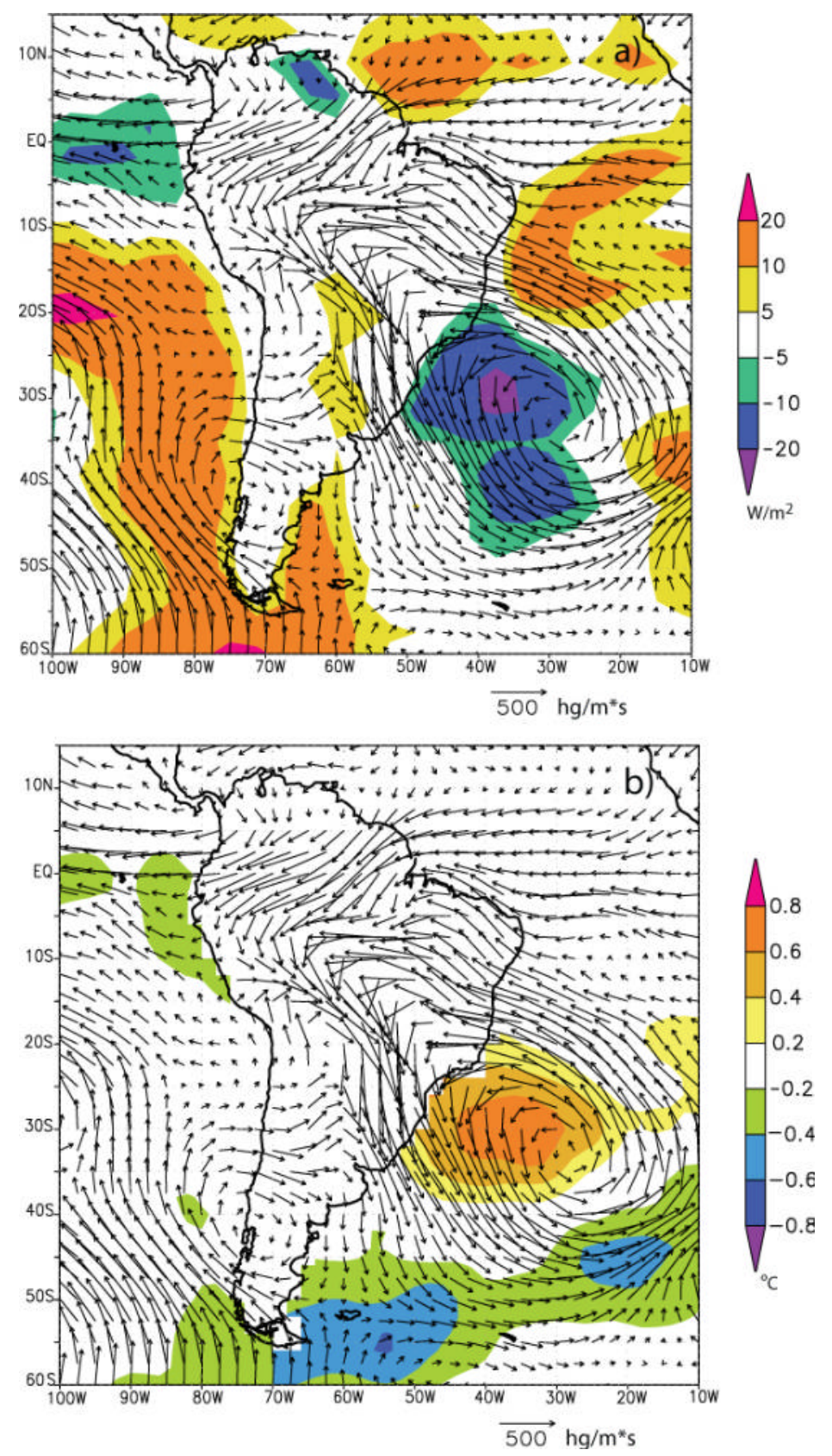

FIG. 7 Composites over events in which the PC of $200 \mathrm{hPa}$ winds is greater than 1 standard deviation for AM of a) latent heat flux anomalies (shaded, $\mathrm{W} / \mathrm{m}^{2}$ ) and vertically integrated moisture flux anomaly (vectors, hg/m*s), and b) SST anomalies (shaded, ${ }^{\circ} \mathrm{C}$ ) and vertically integrated moisture flux anomaly (vectors, hg/m*s). 

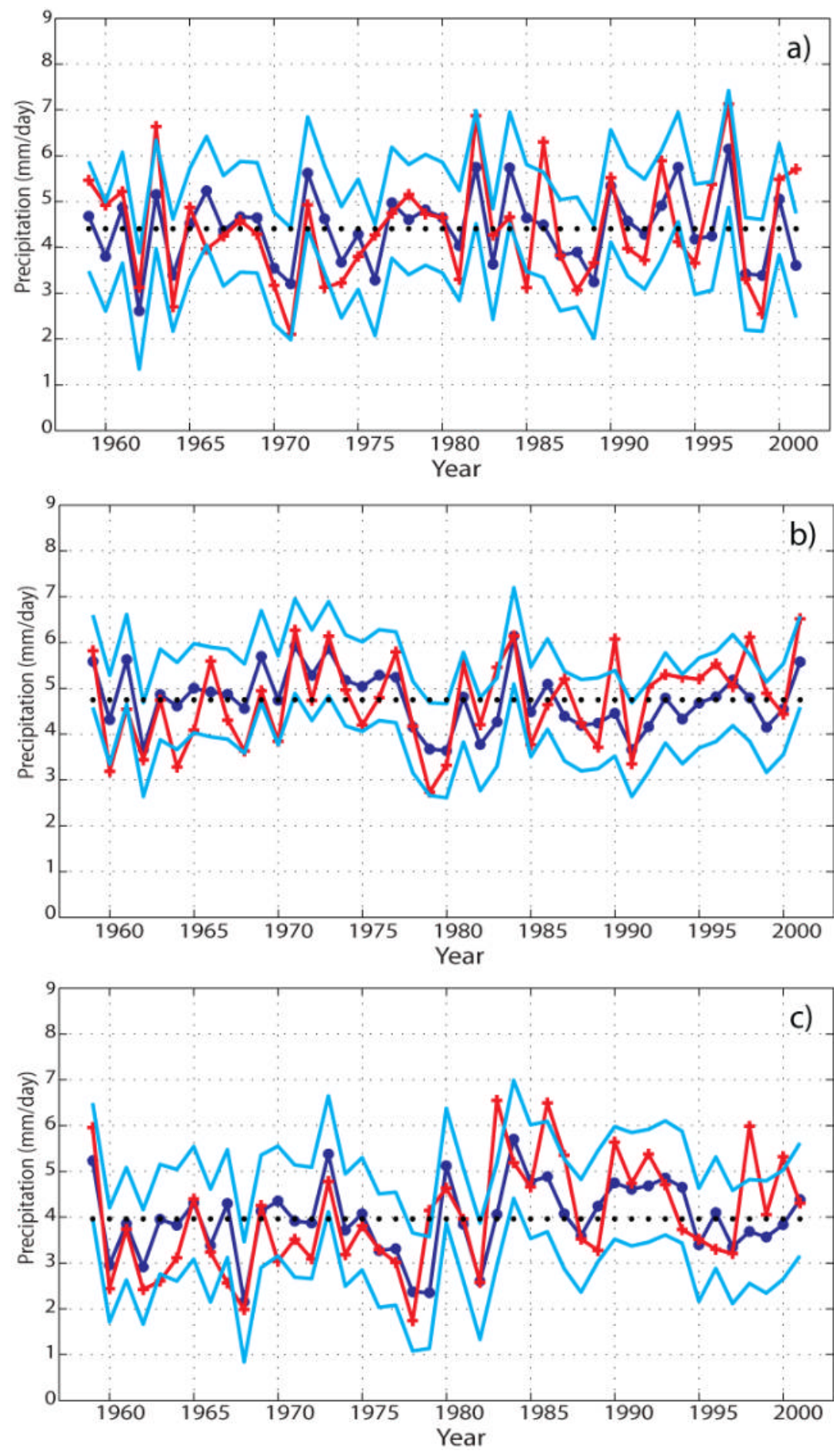

FIG. 8 Potential predictability of precipitation over SESA. Mean of the distribution of the empirical model (blue), $80 \%$ confidence interval (light blve), climatology (dotted), and observations (red) for $\mathrm{ON} \mathrm{a}$ ), b) JF, and c) AM. 

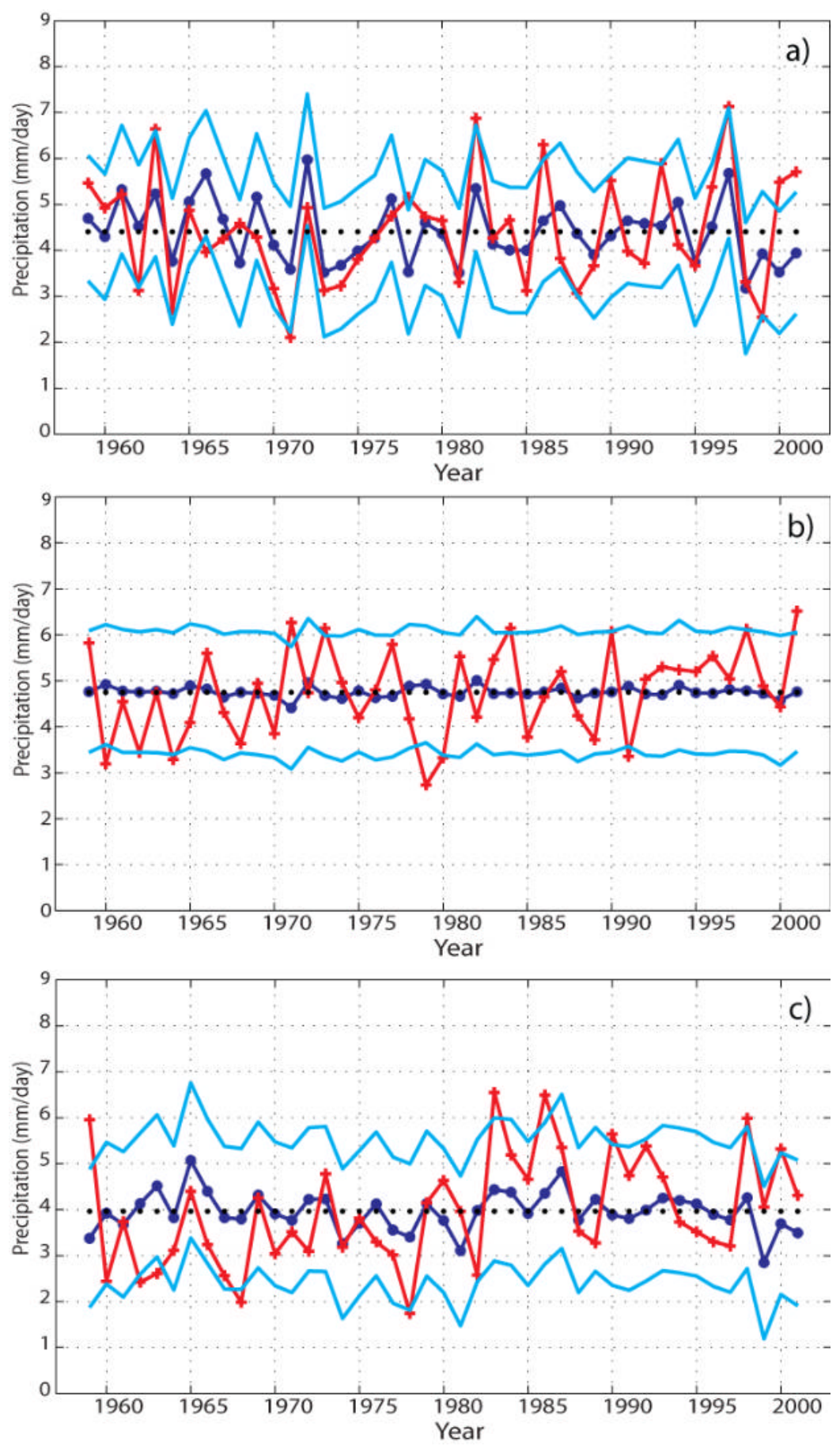

FIG. 9 Experimental forecasts of precipitation over SESA. Mean of the distribution of the empirical model (blue), $80 \%$ confidence interval (light blue), climatology (dotted), and observations (red) for $\mathrm{ON} \mathrm{a}$ ), b) JF, and c) AM. 

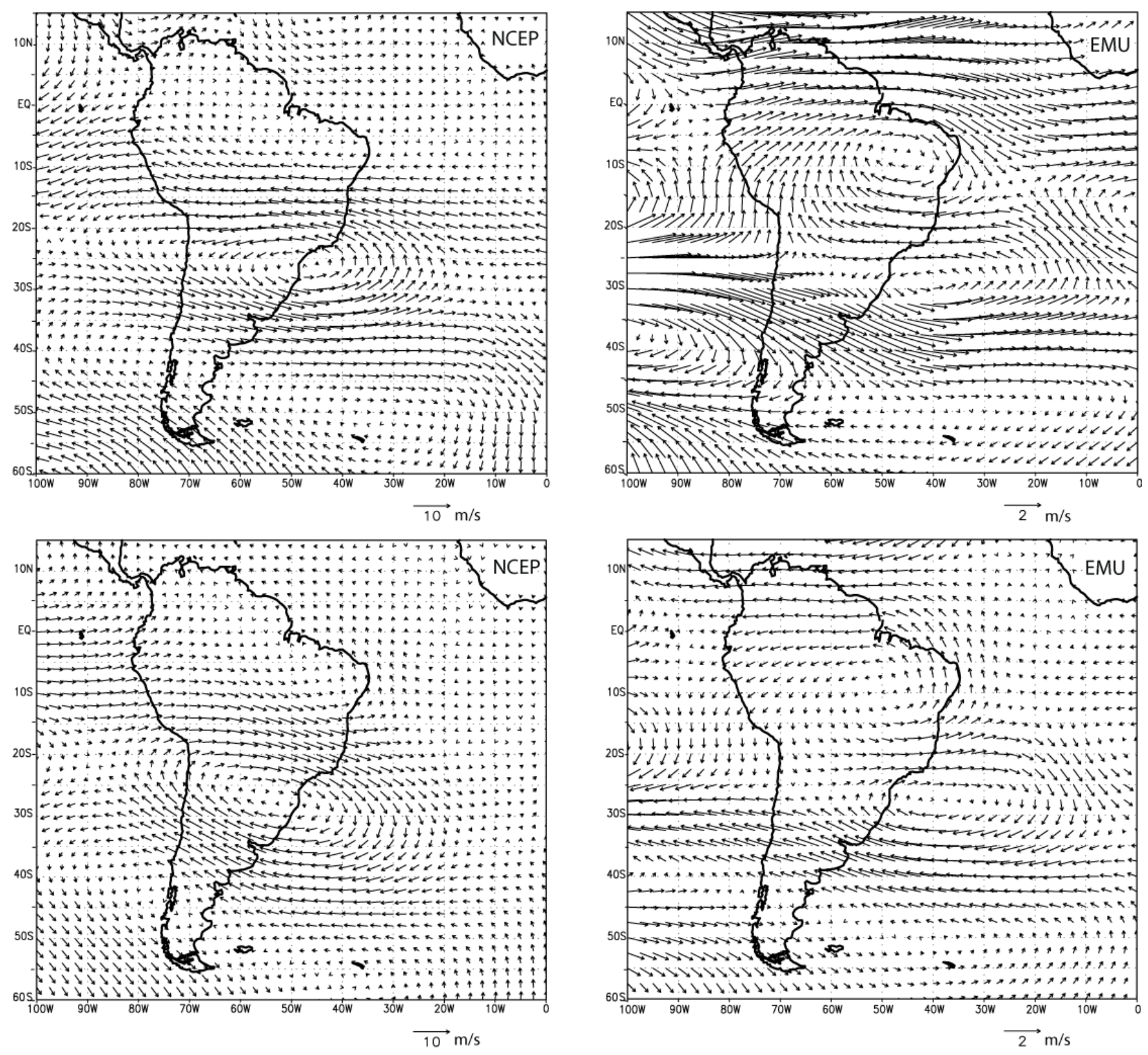

FIG. 10 Composite of $200 \mathrm{hPa}$ winds (m/s) over events in which the PC of $200 \mathrm{hPa}$ winds is greater than 1 standard deviation (upper panels) and smaller than - 1 standard deviation (lower panels) for the NCEP reanalysis (left panels) and EMU (right panels) for ON. 

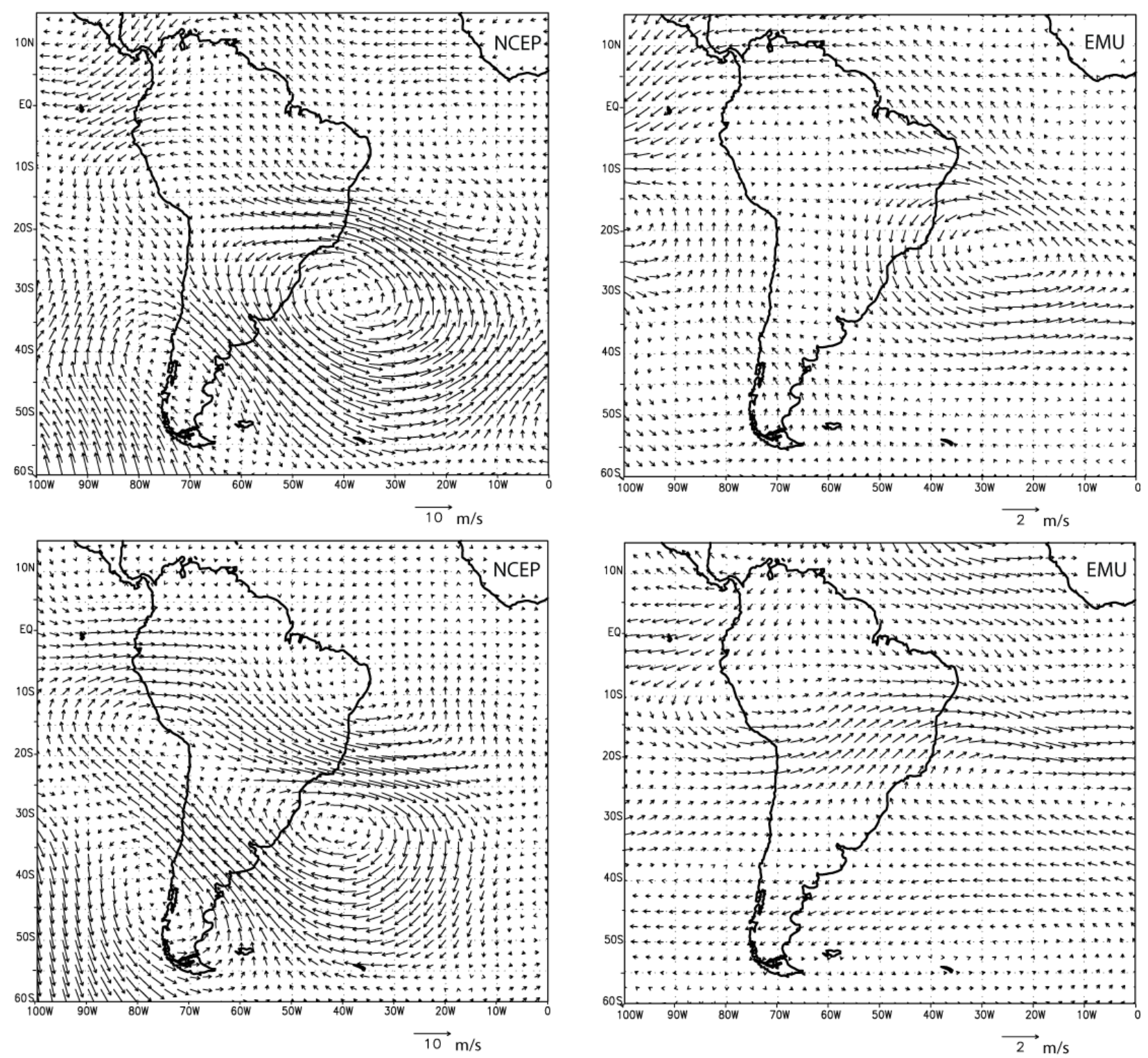

FIG. 11 Same as FIG. 10 but for AM. 


\begin{tabular}{|c|c|c|}
\hline Season & $\begin{array}{c}\text { Skill score of } \\
\text { predictability }\end{array}$ & $\begin{array}{c}\text { Skill score of } \\
\text { experimental } \\
\text { forecasts }\end{array}$ \\
\hline ON & 0.42 & 0.24 \\
\hline JF & 0.41 & -0.08 \\
\hline AM & 0.41 & 0.03 \\
\hline
\end{tabular}

TABLE 1. Skill of the empirical model for the predictability and experimental forecasts (see text for details). Skill is measured by the mean squared error skill score. 


\section{FIGURE CAPTION LIST}

FIG.1 Precipitation (shaded, $\mathrm{mm} /$ day) and vertically integrated moisture flux (vectors, $\mathrm{hg} / \mathrm{m}^{*} \mathrm{~s}$ ) climatology over SA for a) ON, b) JF, c) AM and interannual variability of the mean precipitation over SESA (mm/day) for d) ON and e) AM. The red box in panel a) represents SESA.

FIG. 2 Leading EOF pattern of $200 \mathrm{hPa}$ interannual variability for ON.

FIG. 3 Composite of horizontal wind (m/s) for AM at (from left to right) 200, 500 and 850 $\mathrm{hPa}$ over events in which the PC of $200 \mathrm{hPa}$ winds is greater than 1 standard deviation. FIG. 4 Water budget terms. Upper panels are the composites of precipitation anomalies (shaded, mm/day) and vertically integrated moisture flux anomalies(vectors, hg/m*s) over events in which the PC of $200 \mathrm{hPa}$ winds is a) greater than 1 standard deviation and b) smaller that -1 standard deviation for ON. Bottom panels are the regression of the PC of winds onto c) evaporation ( $\mathrm{mm} /$ day) and d) divergence of the vertically integrated moisture flux (mm/day). Note the scale of the evaporation represents anomalies one order of magnitude smaller compared to the other fields.

FIG. 5 As in FIG. 4 but for JF and C) is the composite of the total vertically integrated moisture flux over events in which the PC of $200 \mathrm{hPa}$ winds is greater than 1 standard deviation.

FIG. 6 As in FIG.4 but for AM.

FIG. 7 Composites over events in which the PC of $200 \mathrm{hPa}$ winds is greater than 1 standard deviation for AM of a) latent heat flux anomalies (shaded, $\mathrm{W} / \mathrm{m}^{2}$ ) and vertically integrated moisture flux anomaly (vectors, $\mathrm{hg} / \mathrm{m}^{*} \mathrm{~s}$ ), and b) SST anomalies (shaded, ${ }^{\circ} \mathrm{C}$ ) and vertically integrated moisture flux anomaly (vectors, $\mathrm{hg} / \mathrm{m} * \mathrm{~s}$ ). 
FIG. 8 Potential predictability of precipitation over SESA. Mean of the distribution of the empirical model (blue), $80 \%$ confidence interval (light blue), climatology (dotted), and observations (red) for ON a), b) JF, and c) AM.

FIG. 9 Experimental forecasts of precipitation over SESA. Mean of the distribution of the empirical model (blue), $80 \%$ confidence interval (light blue), climatology (dotted), and observations (red) for ON a), b) JF, and c) AM.

FIG. 10 Composite of $200 \mathrm{hPa}$ winds (m/s) over events in which the PC of $200 \mathrm{hPa}$ winds is greater than 1 standard deviation (upper panels) and smaller than -1 standard deviation (lower panels) for the NCEP reanalysis (left panels) and EMU (right panels) for ON.

FIG. 11 Same as FIG. 10 but for AM. 